\title{
Safety of Surgical Tracheostomy during Extracorporeal Membrane Oxygenation
}

\author{
Hye Ju Yeo ${ }^{1}$, Seong Hoon Yoon ${ }^{1}$, Seung Eun Lee', Doosoo Jeon ${ }^{1}$, Yun Seong Kim ${ }^{1}$, Woo Hyun Cho' ${ }^{1}$, and Dohyung Kim ${ }^{2}$ \\ 1Department of Pulmonology and Critical Care Medicine, ${ }^{2}$ Department of Thoracic and Cardiovascular Surgery, Pusan National University Yangsan Hospital, \\ Research Institute for Convergence of Biomedical Science and Technology, Yangsan, Korea
}

Background: The risk of bleeding during extracorporeal membrane oxygenation (ECMO) is a potential deterrent in performing tracheostomy at many centers. To evaluate the safety of surgical tracheostomy (ST) in critically ill patients supported by ECMO, we reviewed the clinical correlation between preoperative coagulation status and bleeding complication-related ST during ECMO.

Methods: From April 1, 2012 to March 31, 2016, ST was performed on 38 patients supported by ECMO. We retrospectively reviewed and analyzed the medical records including complications related to ST.

Results: Heparin was administered to 23 patients (60.5\%) for anticoagulation during ECMO, but 15 patients (39.5\%) underwent ECMO without anticoagulation. Of the 23 patients administered anticoagulation therapy, heparin infusion was briefly paused in 13 prior to ST. The median platelet count, international normalized ratio, and activated partial thromboplastin time before ST were $126 \times 10^{9} / \mathrm{L}$ (range, 46 to $434 \times 10^{9} / \mathrm{L}$ ), 1.2 (range, 1 to 2.3 ) and 62 seconds (27 to 114.2 seconds), respectively. No peri-procedural clotting complications related to ECMO were observed. Two patients (5.3\%) suffering from ST-related major bleeding required surgical hemostasis. Minor bleeding after ST occurred in two cases (5.3\%). No significant difference was found according to anticoagulation management (P = 0.723). No fatality was attributable to ST.

Conclusions: The complication rates of ST in the patients supported by ECMO were low. Therefore, ST performed by an experienced operator, and with careful optimization of coagulation status, is a relatively safe procedure; the use of ST with ECMO should thus not be dismissed on account of the potential for bleeding caused by the administration of anticoagulants.

Key Words: anticoagulation; bleeding; extracorporeal membrane oxygenation; tracheostomy.

\section{Introduction}

Most centers carrying out extracorporeal membrane oxygenation (ECMO) find difficulty in performing extubation while patients receive ECMO [1]. Use of ECMO with the patient awake could avoid several disadvantage related to sedation, intubation, and mechanical ventilation [2]. However, given the complexity of patient-ECMO interaction, in practical terms, this method would be applicable only for some patients at centers where personnel are sufficiently experienced [3]. Thus, tracheostomy is recommended when prolonged mechanical ventilation is presumed [4]. However, the risks of bleeding during ECMO may deter many centers from performing surgical tracheostomy (ST). Therefore, it is clinically

\footnotetext{
Received on February 1, 2017 Revised on March 21, 2017 Accepted on March 21, 2017

Correspondence to: Dohyung Kim, Department of Thoracic and Cardiovascular Surgery, Pusan National University Yangsan Hospital, Research Institute for Convergence of Biomedical Science and Technology, 20 Geumo-ro, Mulgeum-eup, Yangsan 50612, Korea

Tel: +82-55-360-2127, Fax: +82-55-360-2157, E-mail: yumccs@nate.com

*No potential conflict of interest relevant to this article was reported.
}

cc This is an Open Access article distributed under the terms of the Creative Commons Attribution Non-Commercial License (http://creativecommons.org/ licenses/by-nc/4.0/) which permits unrestricted non-commercial use, distribution, and reproduction in any medium, provided the original work is properly cited. Copyright (c) 2017 The Korean Society of Critical Care Medicine 
important to evaluate safety issues through a review of adverse events after ST during the use of ECMO. We reviewed and analyzed the clinical correlation of preoperative coagulation status and bleeding complications after ST during ECMO utilization in critically ill patients.

\section{Materials and Methods}

We retrospectively identified all patients who underwent ST while on ECMO support, from April 2012 to March 2016. The following routine clinical baseline data were collected: age, sex, diagnosis and reason for ECMO support, Acute Physiology and Chronic Health Evaluation II score, type of ECMO, time from commencement of mechanical ventilation to start of ECMO support, and time from start of ECMO to tracheostomy. In addition, the following hematological and coagulatory parameters were measured: hemoglobin level, platelet count, international normalized ratio (INR), activated partial thromboplastin time (aPTT) prior to ST, and amount and type of blood products administrated 24 hours before and after ST. The institutional review board of our hospital approved this study and waived the requirement for written informed consent.

\section{1) ECMO protocol and routine care}

We applied the ECMO protocol based on the Extracorporeal Life Support Organization guidelines [5]. The ECMO system consisted of a polymethylpentene fiber oxygenator system (QUADROX PLS; Maquet Inc., Hirrlingen, Germany) with simplified Bioline-coated circuits (Maquet Inc., Rastatt, Germany). All patients were supported with centrifugal pumps (Maquet Inc.), and were cannulated peripherally through the femoral artery and vein or internal jugular vein using the Seldinger technique. Patients received an initial unfractionated heparin (UFH) bolus of 50 units $/ \mathrm{kg}$ body weight when the cannula was placed, and UFH was continuously infused during ECMO. Exceptionally, patients who have bleeding complications supported ECMO without anticoagulation therapy. Based on assessment by the attending physician and intensivist of the individual benefits and risks of the procedure, most patients on ECMO who were undergoing, or expected to undergo, a prolonged weaning process underwent the ST procedure.

\section{2) Anticoagulation and tracheostomy protocol}

Our anticoagulation protocol involved stopping the administration of intravenous UFH 4 hours before the planned tracheostomy. However, we did not stop heparin infusion in patients at high risk of a thromboembolic event. ST was performed at the bedside in the intensive care unit (ICU) under sedation with propofol and remifentanil. The patient's shoulders were elevated and the head extended (unless cervical disease or injury was present), thereby elevating the upper trachea. Sterile drapes were placed, creating a surgical window from the top of the larynx to the suprasternal notch. After administration of local anesthesia, a skin incision $(2-3 \mathrm{~cm})$ was made at two fingers' width from the upper sternal notch. Sharp dissection was then made to cut through the platysma muscle, with bleeding controlled by hemostats and electrocautery. Blunt dissection parallel to the long axis of the trachea was then used to separate the submuscular tissues until the thyroid isthmus was identified. The isthmus must be mobilized and a small incision made to create a space for the tracheostomy procedure. Blunt dissection was continued longitudinally through the pretracheal fascia, and the target tracheal ring (usually the third) was identified. Complete removal of the anterior part of this ring, according to tube size, was performed to create the stoma. After removal of the intubation tube, the tracheostomy tube was inserted with an attached ventilator. With continuous control of bleeding, the tracheostomy was completed. Two hours following the end of the tracheostomy procedure, heparin administration was initiated at the previous rate and adjusted according to subsequent coagulation test results. After surgery, the patients were maintained in a state of wakefulness without sedation. 


\section{3) Outcome definition}

Complications associated with the tracheostomy procedure were graded as either major or minor, and were followed up until either death or hospital discharge. Major complications were defined as any of the following: procedure-related death, acute hypotension, tracheal wall injury, pneumothorax, major bleeding requiring surgical repair, and stoma infection. Minor complications included localized minor bleeding, which was defined as selflimiting bleeding or bleeding successfully treated by local compression. In general, in patients undergoing tracheostomy with ECMO support, oozing-type bleeding frequently occurs, with no special postoperative monitoring focus. We considered this oozing-type bleeding as major if an additional hemostatic procedure, such as electrocauterization, was required. Any clotting complications related to ECMO were recorded, including any documented evidence of visible new clot formation within the circuit or a sudden malfunction of the ECMO within 24 hours of the ST procedure.

\section{4) Statistical analysis}

All statistical analyses were performed using $\mathrm{R}$ software version 3.0.1 (http://www.R-project.org). Continuous variables were described as medians and ranges (minimum to maximum) and compared using the nonparametric Wilcoxon rank sum test. Categorical variables were described as numbers (\%) and compared using Fisher exact test or chi-squared test. All tests were two-sided, and P-values less than 0.050 were considered statistically significant.

\section{Results}

\section{1) Baseline characteristics}

Between April 1, 2012 and March 31, 2016, 38 patients underwent ST while on ECMO. Their median age was 59 years (range, 20 to 83 years), and 26 (68.4\%) were male. Thirty-three patients $(86.8 \%)$ were on venoveno (VV) ECMO; three (7.9\%) were on venovenoarterial
(VVA) ECMO; and two (5.3\%) were on venoarterial (VA) ECMO. The main indication for ECMO was severe acute respiratory distress syndrome, which was present in 31 patients $(81.6 \%)$. The main primary lung-related diagnosis was pneumonia, which was present in 13 patients

Table 1. Baseline characteristics of patients with ST on ECMO

\begin{tabular}{|c|c|}
\hline Variable & Value $(n=38)$ \\
\hline Male sex & $26(68.4)$ \\
\hline Age (yr) & $59(20-83)$ \\
\hline \multicolumn{2}{|l|}{ Reason for ECMO } \\
\hline ARDS & $28(73.7)$ \\
\hline ARDS with RVF & $3(7.9)$ \\
\hline Primary cardiac failure & $7(18.4)$ \\
\hline \multicolumn{2}{|l|}{ Primary diagnosis } \\
\hline Pneumonia & $13(34.2)$ \\
\hline Acute exacerbation of COPD & $1(2.6)$ \\
\hline Acute exacerbation of ILD & $9(23.7)$ \\
\hline Trauma & $1(2.6)$ \\
\hline Postop ARDS & $9(23.7)$ \\
\hline Septic cardiomyopathy & $3(7.9)$ \\
\hline Acute coronary syndrome & $2(5.3)$ \\
\hline APACHE II score & $15.5(6-39)$ \\
\hline \multicolumn{2}{|l|}{ Anticoagulation } \\
\hline Heparin & $23(60.5)$ \\
\hline No heparin & $15(39.5)$ \\
\hline \multicolumn{2}{|l|}{ ECMO mode } \\
\hline W & $33(86.8)$ \\
\hline WA & $3(7.9)$ \\
\hline VA & $2(5.3)$ \\
\hline ST on days after intubation & $9.5(1-25)$ \\
\hline ST on days after ECMO initiation & $9(1-18)$ \\
\hline Early tracheostomy (<8 days of intubation) & $12(31.6)$ \\
\hline Mechanical ventilation day & $33.5(4-76)$ \\
\hline Hospital day & $62.5(14-228)$ \\
\hline ICU day & $38(10-163)$ \\
\hline Successful weaning of ECMO & $25(65.8)$ \\
\hline 6-Month survival & $17(44.7)$ \\
\hline
\end{tabular}

Values are presented as number (\%) or median (range).

ECMO: extracorporeal membrane oxygenation; ARDS: acute respiratory distress syndrome; RVF: right ventricular failure; COPD: chronic obstructive pulmonary disease; ILD: interstitial lung disease; APACHE: Acute Physiology and Chronic Health Evaluation; W: venovenous; WA: venovenoarterial; VA: venoarterial; ST: surgical tracheostomy; ICU: intensive care unit. 
Table 2. Coagulatory parameters before and after ST

\begin{tabular}{|c|c|c|c|c|}
\hline \multirow[b]{2}{*}{ Variable } & \multirow[b]{2}{*}{ Total $(\mathrm{n}=38)$} & \multicolumn{2}{|c|}{ Heparin $(n=23)$} & \multirow{2}{*}{$\begin{array}{l}\text { No-heparin } \\
(\mathrm{n}=15)\end{array}$} \\
\hline & & $\begin{array}{l}\text { Stop the heparin before ST } \\
\qquad(\mathrm{n}=13)\end{array}$ & $\begin{array}{l}\text { Maintain the heparin before ST } \\
\qquad(\mathrm{n}=10)\end{array}$ & \\
\hline \multicolumn{5}{|l|}{ Prior to ST } \\
\hline Hemoglobin ( $\mathrm{g} / \mathrm{dl})$ & $9.6(7.5-12.5)$ & $10.4(7.5-12.2)$ & $9.6(8.0-12.5)$ & $9.5(7.5-11.9)$ \\
\hline Platelets $\left(\times 10^{9} / \mathrm{L}\right)$ & $126(46-434)$ & $127(65-167)$ & $150(46-352)$ & $121(46-434)$ \\
\hline $\mathbb{N N R}$ & $1.2(1-2.3)$ & $1.1(1.1-1.4)$ & $1.1(1-1.3)$ & $1.3(1.1-2.3)$ \\
\hline aPTT (s) & $62(27-114.2)$ & $66.7(37.3-104.5)$ & $74.2(46.2-114.2)$ & $49.4(27-96.4)$ \\
\hline \multicolumn{5}{|l|}{ Within 24 h post-ST } \\
\hline Hemoglobin (g/dl) & $9.4(6.4-12.7)$ & $9.7(6.4-12.2)$ & $9.5(8.0-12.5)$ & $9.2(7.8-12.3)$ \\
\hline
\end{tabular}

Values are presented as median (range).

ST: surgical tracheostomy; INR: international normalized ratio; aPTT: activated partial thromboplastin time.

(34.2\%). Twenty-three patients $(60.5 \%)$ were administered UFH for anticoagulation during ECMO, while 15 patients were supported by ECMO without anticoagulation due to bleeding complications prior to ST. The most common site of bleeding was retroperitoneal (5/15), followed by gastrointestinal (4/15), intrapulmonary (4/15), and cannula site $(2 / 15)$. ST was performed on a median of 9.5 days after intubation (range, 1 to 25 days) and on a median of 9 days after ECMO initiation (range, 1 to 18 days). Early tracheostomy ( $<8$ days after intubation) was performed in 12 cases $(31.6 \%)$ (Table 1$)$.

\section{2) Coagulation characteristics}

The median values for hemoglobin, platelet count, INR, and aPTT before ST were $9.6 \mathrm{~g} / \mathrm{dl}$ (range, 7.5 to $12.5 \mathrm{~g} / \mathrm{dl}$ ), $126 \times 10^{9} / \mathrm{L}$ (range, 46 to $434 \times 10^{9} / \mathrm{L}$ ), 1.2 (range, 1 to 2.3 ), and 62 seconds (27 to 114.2 seconds), respectively. The median values for hemoglobin in the 24 hours following ST were similar to those during the previous 24 hours (median, $9.4 \mathrm{~g} / \mathrm{dl}$; range, 6.4 to $12.7 \mathrm{~g} / \mathrm{dl}$ ). In 13 out of the 23 patients administered anticoagulation therapy, heparin infusion was paused before ST, while in 10 it was maintained during the procedure. We classified patients into three groups in terms of pre-tracheostomy heparin management (group 1, heparin stopped within 4 hours before ST; group 2, heparin maintained during ST; group 3 , no heparin administered before ST). There were no significant differences among the three groups regarding
Table 3. ST-related complications

\begin{tabular}{lc}
\hline Complication & Value \\
\hline ST-related death & 0 \\
Acute hypotension & 0 \\
Major bleeding & 2 \\
Minor bleeding & 2 \\
Pneumothorax & 0 \\
Tracheal wall injury & 0 \\
Stromal infection & 0 \\
ECMO clotting post-ST & 0 \\
\hline
\end{tabular}

ST: surgical tracheostomy; ECMO: extracorporeal membrane oxygenation.

the median values for hemoglobin and platelet count before ST $(\mathrm{P}=0.796, \mathrm{P}=0.579)$. However, there were significant differences among the three groups regarding the median values for INR and aPTT before ST $(\mathrm{P}=0.020, \mathrm{P}$ $=0.014)$. In addition, there were no significant differences among the three groups regarding the median values for hemoglobin 24 hours after ST $(\mathrm{P}=0.166)$ (Table 2$)$.

\section{3) Complications}

There were no ST procedure-related deaths or periprocedural thrombotic complications related to ECMO. Two patients $(5.3 \%)$ suffered a major bleeding complication: one patient on VV ECMO, and for whom the heparin infusion was stopped before ST, developed bleeding at the tracheostomy site, which required electrocauterization; the second patient, supported by VA ECMO and not ad- 
ministered heparin due to retroperitoneal bleeding, developed delayed postoperative bleeding from a small vessel arising from the innominate artery. This was due to vascular erosion of the friable mucosa as a result of friction from the tracheostomy tube, and required surgical repair. All other complications were classified as minor bleeding $(\mathrm{n}=2,5.3 \%)$ and were treated with local compression.

Regarding the incidence of major or minor bleeding, there was no significant difference found among three groups according to pre-tracheostomy heparin management $(\mathrm{P}=0.723)$. The total bleeding incidence after ST in groups 1,2 , and 3 was $15.4 \%, 0 \%$, and $13.3 \%$, respectively (Table 3 ).

\section{4) Clinical course and further outcomes}

The successful rates of weaning off ECMO and 6 -month survival were $65.8 \%$ and $44.7 \%$, respectively. The total durations of mechanical ventilation, ECMO support, and ICU/hospital length of stay are presented in Table 1. There were no significant differences between the early ( $<8$ days of intubation) and late tracheostomy groups regarding both 6-month survival rates $(41.7 \%$ and $46.2 \%$, respectively; $\mathrm{P}=0.796$ ) and median hospital length of stay (57.5 days [range, 14 to 149 days] and 65 [range, 16 to 228], respectively, $\mathrm{P}=0.343$ ). However, early tracheostomy significantly reduced the median number of days on mechanical ventilation (23.5 days [range, 4 to 63 days] and 38.5 days [range, 14 to 76 days], respectively, $\mathrm{P}=0.007$ ) and median ICU length of stay (27 days [range, 10 to 63 days] and 47 days [range, 14 to 163 days], respectively, $\mathrm{P}=0.013$ ) (Figure 1). In addition, the median number of days ventilator-free [6] was significantly higher in the early than the late tracheostomy group (4.5 days [range, 0 to 24 days] and 0 day [range, 0 to 14 days], respectively, $\mathrm{P}=0.040$ ).

\section{Discussion}

Of the 38 patients who underwent ST while on ECMO, the overall rate of ST-related clinically relevant complications was $10.6 \%$ and the rate of major bleeding complications was $5.3 \%$. Our results suggest that ST can be safely used by experienced operators on adult patients undergoing ECMO, with careful optimization of the coagulation status.

Utilization of ECMO in the awake patient in the ICU has gained widespread acceptance [2]. However, despite the inherent benefits, extubation during ECMO is possible only for some patients and under the control of experienced personnel. Camporota et al. [1] reported that very few centers extubated patients during ECMO (almost

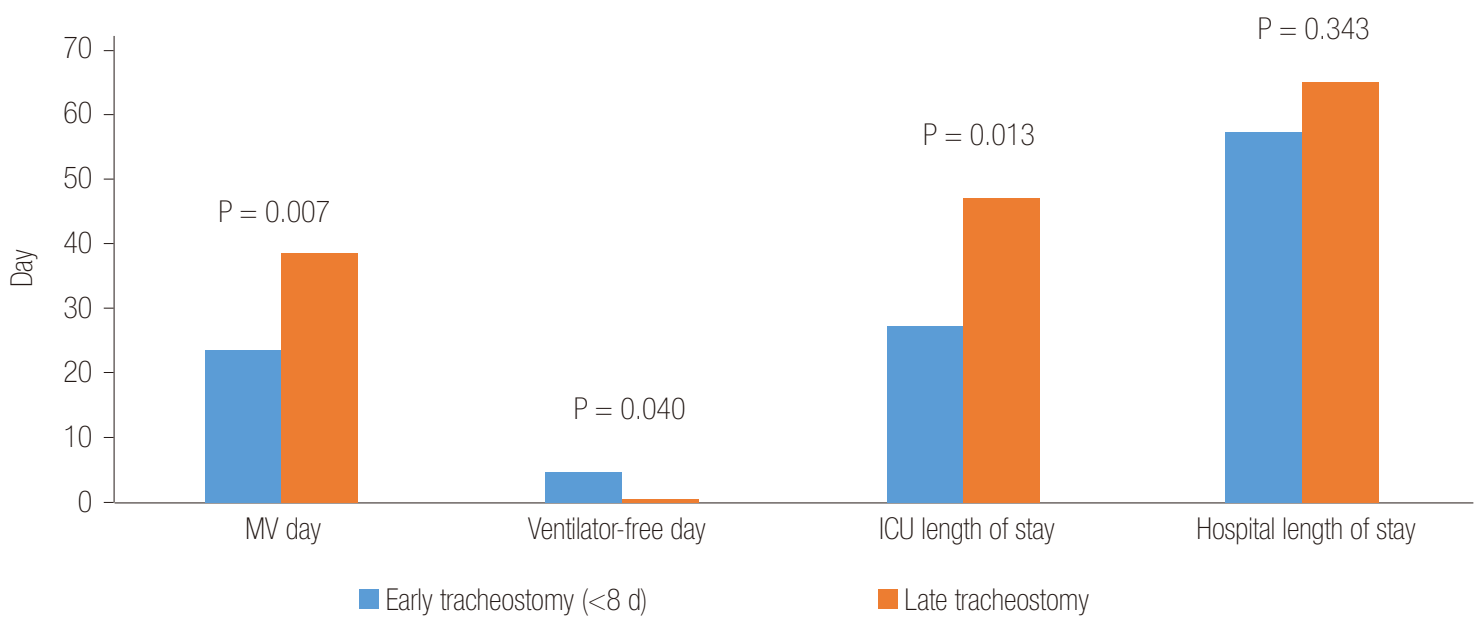

Figure 1. Differences in clinical outcomes between early and late tracheostomy in patients on extracorporeal membrane oxygenation. Values are presented as the median day. MV: mechanical ventilator; ICU: intensive care unit. 
$90 \%$ of centers extubated $<25 \%$ of their patients). Only $1.5 \%$ of the ECMO centers reviewed extubated more than $75 \%$ of their patients while on ECMO. Currently, most clinicians prefer to keep patients intubated or perform an early tracheostomy to allow self-ventilation.

The most important clinical benefit of tracheostomy is to allow early physical therapy and active rehabilitation by avoiding sedation and paralytics. It also facilitates pulmonary toilet and early ventilator weaning, and intermittent mechanical ventilation to help prevent atelectasis, especially when the patient is fatigued after active rehabilitation [7,8]. Despite the fact that tracheostomy is required in many patients supported by ECMO for respiratory support, almost $30 \%$ of ECMO centers never perform tracheostomy on patients with ECMO, in view of the increased risk of bleeding [1].

Hemorrhage is a potential complication that can be significantly exacerbated by the use of anticoagulation which is required with ECMO, and may thus have a marked impact on the patient's further clinical course $[3,9]$. The degree of bleeding may be increased either during or after tracheostomy in patients supported by ECMO, due to the activation of coagulation enzymes, or to platelet depletion or anticoagulant medication [10].

The rates of major bleeding in our study are similar to those recorded in studies on ST in critically ill patients [11-13]. Costa et al. [13] reported major complications in $12.2 \%$ of 56 patients undergoing ST in the emergency department. Bleeding was the most frequent complication, although no deaths were related to the procedure. Schmidt et al. [3] reported no fatal complications directly related to ST in patients supported by ECMO. In that study, bleeding was the most frequent complication of ST and no significant difference was noted regarding the type of anticoagulation management, e.g., heparin cessation or maintenance. It remains to be established whether the use of anticoagulation increases the incidence of bleeding during tracheostomy in patients on ECMO. Pasin et al. [14] reported a minor bleeding incidence of $19 \%$ for tracheostomy, and an incidence of $6 \%$ for major bleeding requiring that transfusion patients be administered anticoagulation. However, in our experience, bleeding complications were not seen in the group administered heparin. This finding may be related to the fact that, in our patients in the heparin group, the therapeutic levels were maintained well by following a low-dose anticoagulation protocol. Previously, we reported that the low-dose heparin strategy is a safe and effective protocol to reduce bleeding in adults undergoing ECMO [15]. Therefore, for the safe performance of tracheostomy, we recommend the use of low-dose heparin during ECMO and for stopping the administration of intravenous UFH 4 hours before the planned tracheostomy. Heparin administration, at the previous rate, should be initiated within 2 hours of completion of the tracheostomy, and adjusted according to subsequent coagulation test results. Exceptionally, in a patient at high risk of a thromboembolic event, ST can be performed while anticoagulation therapy is administered. In such a case, the surgeon(s) must be skilled in performing proper and accurate hemostasis to prevent postoperative bleeding.

Recently, percutaneous dilatational tracheostomy (PDT) has replaced ST in many ICUs, with comparable safety and convenience in the hands of the experienced operator [16,17]. Although PDT is more accessible and less timedemanding compared to ST, it has several weaknesses [18-21]. PDT is associated with a high risk of both morbidity and mortality in certain patients, and includes the requirement for high positive end-expiratory pressure or fraction of inspired oxygen $\left(\mathrm{FiO}_{2}\right)$, difficult anatomy (e.g., obesity, thick short neck, or tracheal deviation), coagulopathy, emergency procedures, and hemodynamic instability $[22,23]$. The need for better visualization of local neck anatomy and improved control of oxygenic/ hemodynamic stability establishes ST as the safest method in critical situations such as ECMO. Although the bleeding incidence is comparable in ST and PDT, the former method is more effective in the prevention and control of bleeding complications $[19,24]$. Several recent reports have been published on the safety of thoracic surgical procedures involving ECMO, but none to date on the safety aspects of ST in patients supported by ECMO 
$[25,26]$. In our experience there were minimal complications, which was easily corrected. ST was well tolerated and enhanced the delivery of care in the period prior to lung recovery.

This study has several limitations. The interpretation of the result is limited by potential bias introduced by the retrospective study design. The available data are limited to those recorded in the medical record, which may have been missed or underestimated. However, we recorded all severe complications, and these were detected by the detailed review. In addition, the small sample size reduces the power of this study. Therefore, further larger studies are needed to confirm these preliminary results and bleeding risk stratification for scheduled ST in patients supported by ECMO.

In conclusion, the complication rates of $\mathrm{ST}$ in patients supported by ECMO were low and comparable to those of other critically ill patients. Therefore, ST during ECMO support is a relatively safe procedure, which should be performed by experienced operators under careful optimization of the coagulation status. The use of ST with ECMO should therefore not be dismissed on account of the potential bleeding complications caused by the administration of anticoagulation.

\section{ORCID}

Hye Ju Yeo

http://orcid.org/0000-0002-8403-5790

\section{References}

1. Camporota L, Nicoletti E, Malafronte M, De Neef M, Mongelli V, Calderazzo MA, et al. International survey on the management of mechanical ventilation during ECMO in adults with severe respiratory failure. Minerva Anestesiol 2015;81:1170-83.

2. Langer T, Santini A, Bottino N, Crotti S, Batchinsky AI, Pesenti A, et al. "Awake" extracorporeal membrane oxygenation (ECMO): pathophysiology, tech- nical considerations, and clinical pioneering. Crit Care 2016;20:150.

3. Schmidt M, Stewart C, Bailey M, Nieszkowska A, Kelly J, Murphy L, et al. Mechanical ventilation management during extracorporeal membrane oxygenation for acute respiratory distress syndrome: a retrospective international multicenter study. Crit Care Med 2015;43:654-64.

4. Keeping A. Early versus late tracheostomy for critically ill patients: a clinical evidence synopsis of a recent Cochrane Review. Can J Respir Ther 2016;52:27-8.

5. Extracorporeal Life Support Organization. Extracorporeal Life Support Organization (ELSO) general guidelines for all ECLS cases [Internet]. Ann Arbor (MI): ELSO; 2013 [cited 2017 May 22]. Available from: https://www.elso.org/Portals/0/IGD/Archive/Fi leManager/929122ae88cusersshyerdocumentselsogu idelinesgeneralalleclsversion1.3.pdf.

6. Schoenfeld DA, Bernard GR; ARDS Network. Statistical evaluation of ventilator-free days as an efficacy measure in clinical trials of treatments for acute respiratory distress syndrome. Crit Care Med 2002;30:1772-7.

7. Hayes D Jr, Galantowicz M, Preston TJ, Lloyd EA, Tobias JD, McConnell PI. Tracheostomy in adolescent patients bridged to lung transplantation with ambulatory venovenous extracorporeal membrane oxygenation. J Artif Organs 2014;17:103-5.

8. Turković TM, Lukić A, Pažur I, Ožegić O, Obraz $M$. The impact of tracheotomy on the clinical course of ventilator-associated pneumonia. Acta Clin Croat 2016;55:100-9.

9. Braune S, Kienast S, Hadem J, Wiesner O, Wichmann D, Nierhaus A, et al. Safety of percutaneous dilatational tracheostomy in patients on extracorporeal lung support. Intensive Care Med 2013;39:1792-9.

10. Malfertheiner MV, Philipp A, Lubnow M, Zeman F, Enger TB, Bein T, et al. Hemostatic changes during extracorporeal membrane oxygenation: a prospective randomized clinical trial comparing three different 
extracorporeal membrane oxygenation systems. Crit Care Med 2016;44:747-54.

11. Shah RK, Lander L, Berry JG, Nussenbaum B, Merati A, Roberson DW. Tracheotomy outcomes and complications: a national perspective. Laryngoscope 2012;122:25-9.

12. Goldenberg D, Ari EG, Golz A, Danino J, Netzer A, Joachims HZ. Tracheotomy complications: a retrospective study of 1130 cases. Otolaryngol Head Neck Surg 2000;123:495-500.

13. Costa L, Matos R, Júlio S, Vales F, Santos M. Urgent tracheostomy: four-year experience in a tertiary hospital. World J Emerg Med 2016;7:227-30.

14. Pasin L, Frati E, Cabrini L, Landoni G, Nardelli P, Bove T, et al. Percutaneous tracheostomy in patients on anticoagulants. Ann Card Anaesth 2015;18:32934.

15. Yeo HJ, Kim do H, Jeon D, Kim YS, Cho WH. Low-dose heparin during extracorporeal membrane oxygenation treatment in adults. Intensive Care Med 2015;41:2020-1.

16. Freeman BD, Isabella K, Lin N, Buchman TG. A meta-analysis of prospective trials comparing percutaneous and surgical tracheostomy in critically ill patients. Chest 2000;118:1412-8.

17. Delaney A, Bagshaw SM, Nalos M. Percutaneous dilatational tracheostomy versus surgical tracheostomy in critically ill patients: a systematic review and meta-analysis. Crit Care 2006;10:R55.

18. Hashemian SM, Digaleh H. A prospective randomized study comparing mini-surgical percutaneous dilatational tracheostomy with surgical and classical percutaneous tracheostomy: a new method beyond contraindications. Medicine (Baltimore) 2015;94:e2015.
19. Johnson-Obaseki S, Veljkovic A, Javidnia H. Complication rates of open surgical versus percutaneous tracheostomy in critically ill patients. Laryngoscope 2016;126:2459-67.

20. Lother A, Wengenmayer T, Benk C, Bode C, Staudacher DL. Fatal air embolism as complication of percutaneous dilatational tracheostomy on venovenous extracorporeal membrane oxygenation, two case reports. J Cardiothorac Surg 2016;11:102.

21. Klancir T, Adam VN, Mršić V, Marin D, Goranović T. Bilateral pneumothorax as a complication of percutaneous tracheostomy: case report. Acta Clin Croat 2016;55 Suppl 1:98-102.

22. Huang CS, Chen PT, Cheng SH, Chen CK, Hsu PK, Hsieh CC, et al. Relative contraindications for percutaneous tracheostomy: from the surgeons' perspective. Surg Today 2014;44:107-14.

23. Lin JC, Maley RH Jr, Landreneau RJ. Extensive posterior-lateral tracheal laceration complicating percutaneous dilational tracheostomy. Ann Thorac Surg 2000;70:1194-6.

24. Brass P, Hellmich M, Ladra A, Ladra J, Wrzosek A. Percutaneous techniques versus surgical techniques for tracheostomy. Cochrane Database Syst Rev 2016;7:CD008045.

25. Redwan B, Ziegeler S, Freermann S, Nique L, Semik $\mathrm{M}$, Lavae-Mokhtari M, et al. Intraoperative venovenous extracorporeal lung support in thoracic surgery: a single-centre experience. Interact Cardiovasc Thorac Surg 2015;21:766-72.

26. Rinieri P, Peillon C, Bessou JP, Veber B, Falcoz PE, Melki J, et al. National review of use of extracorporeal membrane oxygenation as respiratory support in thoracic surgery excluding lung transplantation. Eur J Cardiothorac Surg 2015;47:87-94. 